\title{
Layer solutions for a class of semilinear elliptic equations involving fractional Laplacians
}

Yan $\mathrm{Hu}^{*}$

\section{"Correspondence:}

huyan1111@126.com

College of Mathematics and

Econometrics, Hunan University,

Changsha, 410082, China

\begin{abstract}
This paper is concerned with the nonlinear equation involving the fractional Laplacian: $(-\triangle)^{s} v(x)=b(x) f(v(x)), x \in \mathbb{R}$, where $s \in(0,1), b: \mathbb{R} \rightarrow \mathbb{R}$ is a periodic, positive, even function and $-f$ is the derivative of a double-well potential $G$. That is, $G \in C^{2, \gamma}(0<\gamma<1), G(1)=G(-1)<G(\tau) \forall \tau \in(-1,1), G^{\prime}(-1)=G^{\prime}(1)=0$. We show the existence of layer solutions of the equation for $s \geq \frac{1}{2}$ and for some odd nonlinearities by variational methods, which is a bounded solution having the limits \pm 1 at $\pm \infty$. Asymptotic estimates for layer solutions as $|x| \rightarrow+\infty$ and the asymptotic behavior of them as $s \uparrow 1$ are also obtained.
\end{abstract}

MSC: 35B20; 35B40; 49J45; 82B26

Keywords: fractional Laplacian; layer solutions; existence; local minimizers

\section{Introduction}

In this paper we study the fractional Laplacian

$$
(-\triangle)^{s} v(x)=b(x) f(v(x)), \quad x \in \mathbb{R}
$$

where $s \in(0,1)$, and $(-\triangle)^{s}$ is the fractional Laplacian defined by

$$
(-\triangle)^{s} v=C_{s} \text { P.V. } \int_{\mathbb{R}} \frac{v(x)-v(y)}{|x-y|^{1+2 s}} d y .
$$

Here P.V. stands for the Cauchy principle value and $C_{s}$ is a positive constant multiplier depending only on $s$.

The fractional Laplacian is a nonlocal operator which can be localized as

$$
\begin{cases}-\operatorname{div}\left(y^{a} \nabla u\right)=0 & \text { in } \mathbb{R}_{+}^{2}, \\ -\lim _{y \downarrow 0^{+}} y^{a} \frac{\partial u}{\partial y}=\frac{1}{d_{s}} b(x) f(u) & \text { on } \partial \mathbb{R}_{+}^{2},\end{cases}
$$

where $a=1-2 s \in(-1,1), d_{s}=2^{2 s-1} \frac{\Gamma(s)}{\Gamma(1-s)}$ and $u(x, 0)=v(x)$. Moreover $u(\cdot, \cdot)$ can be expressed by a Poisson kernel,

$$
u(x, y)=P_{s}(\cdot, y) * v=p_{s} \int_{\mathbb{R}} \frac{y^{2 s}}{\left(|z|^{2}+y^{2}\right)^{\frac{1+2 s}{2}}} v(x-z) d z \quad \text { for every } y>0,
$$

○2014 Hu; licensee Springer. This is an Open Access article distributed under the terms of the Creative Commons Attribution License (http://creativecommons.org/licenses/by/2.0), which permits unrestricted use, distribution, and reproduction in any medium, provided the original work is properly cited. 
which is called the $s$-extension of $v \cdot p_{s}$ is a positive constant depending only on $s$. For more details as regards the fractional Laplacian, readers can refer to [1-7] and the references therein.

In view of the celebrated De Giorgi conjecture (see [8-10]), Cabré and Sire [2, 3] considered layer solutions of the nonlocal equation

$$
(-\triangle)^{s} v=f(v) \quad \text { in } \mathbb{R}
$$

The necessary and sufficient conditions for the existence of one-dimensional layer solutions were given as

$$
G(1)=G(-1)<G(s) \quad \forall s \in(-1,1), \quad G^{\prime}(1)=G^{\prime}(-1)=0,
$$

where $G^{\prime}=-f$. All these were obtained by a Hamiltonian equality and a Modica-type estimate for layer solutions. By the sliding method, the layer solution of (1.3) was proved to be the unique local minimizer which increases in $x$ with values varying from -1 to 1 . The regularity, Hopf principle, maximum principle as well as a Harnack inequality for (1.3) or for its extension equation (1.2) (in this case $b=1$ ) were given. Some of them will be used in our paper.

If $b$ is not a constant and is periodic, the perturbed equation (1.1) becomes complicated. The aim of this paper is to study the layer solution of (1.1) with periodic perturbed nonlinearity.

Definition 1.1 A function $v \in\left(L^{\infty} \cap C^{\beta}\right)(\mathbb{R})(0<\beta<1)$ is said to be a layer solution of (1.1), if $v$ solves (1.1),

$$
(-\triangle)^{s} v(x)=b(x) f(v(x)), \quad x \in \mathbb{R}
$$

and

$$
\lim _{x \rightarrow \pm \infty} v(x)= \pm 1
$$

Definition 1.2 A function $u \in L^{\infty}\left(\mathbb{R}_{+}^{2}\right) \cap C^{\beta}\left(\overline{\mathbb{R}_{+}^{2}}\right)$ is said to be a layer solution of (1.2), if $u$ solves (1.2),

$$
\begin{cases}-\operatorname{div}\left(y^{a} \nabla u\right)=0 & \text { in } \mathbb{R}_{+}^{2}, \\ -\lim _{y \downarrow 0^{+}} y^{a} \frac{\partial u}{\partial y}=\frac{1}{d_{s}} b(x) f(u) & \text { on } \partial \mathbb{R}_{+}^{2}\end{cases}
$$

and

$$
\lim _{x \rightarrow \pm \infty} u(x, 0)= \pm 1
$$

Namely, $u(x, 0)$ is the corresponding layer solution of $(1.1)$.

Different from the unperturbed case (1.3), the inhomogeneous term $b(x) f(u)$ depends explicitly on $x$ in (1.1) and (1.2); the sliding method cannot be used and layer solutions of them have no monotonicity in the direction of $x$. The method for obtaining layer solutions in [2] and [3] cannot be used in our case directly; some difficulties need to be solved. 
In the paper, we consider the extension problem (1.2). Obviously, (1.2) has a variational structure.

Denote

$$
\begin{aligned}
& \Omega \subset \mathbb{R}_{+}^{2}, \text { a bounded Lipschitz domain, } \\
& B_{R}(x, y) \subset \mathbb{R}^{2} \text {, a ball centered at }(x, y) \in \mathbb{R}^{2} \text { with radius } R, \\
& B_{\epsilon}^{+}(x, 0)=B_{\epsilon}(x, 0) \cap \mathbb{R}_{+}^{2}, \\
& \partial^{0} \Omega=\left\{(x, 0) \in \partial \Omega \cap \partial \mathbb{R}_{+}^{2} \mid \exists \epsilon>0, B_{\epsilon}^{+}(x, 0) \subset \Omega\right\}, \\
& \partial^{+} \Omega=\overline{\partial \Omega \cap \mathbb{R}_{+}^{2}} .
\end{aligned}
$$

For $u \in H^{1}\left(y^{a}, \Omega\right)$, the norm is

$$
\|u\|_{H^{1}\left(y^{a}, \Omega\right)}=\left(\int_{\Omega} y^{a}|\nabla u|^{2} d x d y\right)^{\frac{1}{2}}+\left(\int_{\Omega} y^{a}|u|^{2} d x d y\right)^{\frac{1}{2}} .
$$

The energy functional of $u$ on $\Omega$ is given by

$$
\mathcal{E}(u, \Omega)=d_{s} \int_{\Omega} \frac{y^{a}}{2}|\nabla u|^{2} d x d y+\int_{\partial^{0} \Omega} b(x) G(u(x, 0)) d x .
$$

We state our main results in the following.

We show, via a Liouville result, the existence of layer solutions of (1.1) for $s \geq \frac{1}{2}$ and for some odd nonlinearities.

Theorem 1.1 Let $s \geq \frac{1}{2}$. Assume that $b, f \in C^{1, \gamma}(\mathbb{R})(0<\gamma<1)$ :

(1) $b: \mathbb{R} \rightarrow \mathbb{R}$ is 1-periodic, even, not constant and positive; denote $\bar{b}=\max _{R} b$ and $\underline{b}=\min _{R} b$

(2) $f(-\tau)=-f(\tau)$ for any $\tau \in[-1,1], f(-1)=f(1)=f(0)=0, f>0$ in $(0,1)$ and $f<0$ in $(-1,0)$.

Obviously, if $G^{\prime}=-f$,

$$
G(-1)=G(1)<G(\tau) \quad \text { for } \tau \in(-1,1), \quad G^{\prime}(1)=G^{\prime}(-1)=0 .
$$

There exists a layer solution $v \in C^{2, \beta}(R)$ (for some $\left.0<\beta<1\right)$ of $(1.1)$ :

$$
\begin{cases}\left(-\partial_{x x}\right)^{s} v(x)=b(x) f(v(x)) & \text { in } \mathbb{R}, \\ v \rightarrow \pm 1 & \text { as } x \rightarrow \pm \infty\end{cases}
$$

In addition, $v$ is odd.

Furthermore we obtain asymptotic estimates of the layer solutions of (1.1) by comparing with a layer solution of the unperturbed equation (1.3).

Theorem 1.2 Let $b \in C^{1, \gamma} \cap L^{\infty}$ is positive. Let $f \in C^{1, \gamma}(\mathbb{R})(\gamma>\max (0,1-2 s))$ satisfy

(i) $G(-1)=G(1)<G(\tau)$ for $\tau \in(-1,1), G^{\prime}(1)=G^{\prime}(-1)=0$;

(ii) $G^{\prime \prime}(1)>0, G^{\prime \prime}(-1)>0$. 
If $v$ is a layer solution of (1.1), then the following asymptotic estimates hold:

$$
\begin{aligned}
& c x^{-2 s} \leq|1-v| \leq C x^{-2 s} \quad \text { for } x>1, \\
& c|x|^{-2 s} \leq|1+v| \leq C|x|^{-2 s} \quad \text { for } x<-1
\end{aligned}
$$

for some constants $0<c<C$.

Finally we investigate the asymptotic behavior of $v^{s}$ as $s \uparrow 1$ and obtain a local elliptic equation, which is stated as follows.

Theorem 1.3 Let $s \in\left[\frac{1}{2}, 1\right)$. Let $\left\{v^{s_{k}}\right\}$ be a sequence of layer solutions of $(1.1)$ in Theorem 1.1. Then, there exists a subsequence denoted again by $\left\{v^{s_{k}}\right\}$ converging locally uniformly to a function $v^{1} \in C^{2}(R)$ as $s_{k} \uparrow 1$, which is also a layer solution of the local elliptic equation

$$
\left\{\begin{array}{l}
-v_{x x}^{1}(x)=b(x) f\left(v^{1}(x)\right) \quad \text { in } \mathbb{R} \\
\lim _{x \rightarrow \pm \infty} v^{1}(x)= \pm 1
\end{array}\right.
$$

In addition,

$$
\frac{1}{2}\left(v_{x}^{1}\right)^{2}=b(x)\left\{G\left(v^{1}(x)\right)-G(1)\right\}+\int_{x}^{+\infty} b^{\prime}(t)\left\{G\left(v^{1}(t)\right)-G(1)\right\} d t .
$$

For convenience of the presentation we will use $C$ for a general positive constant; such a $C$ is usually different in different contexts.

\section{Some preliminaries and properties}

In this paper, we mainly study the extension equation (1.2). To make our problems clear, we present several properties of layer solutions.

Lemma 2.1 Let $u$ be a bounded solution of (1.2),

$$
\begin{cases}-\operatorname{div}\left(y^{a} \nabla u\right)=0 & \text { in } \mathbb{R}_{+}^{2}, \\ -\lim _{y \downarrow 0^{+}} y^{a} \frac{\partial u}{\partial y}=\frac{1}{d_{s}} b(x) f(u) & \text { on } \partial \mathbb{R}_{+}^{2}\end{cases}
$$

and

$$
\lim _{x \rightarrow \pm \infty} u(x, 0)=L^{ \pm}
$$

with two constants $L^{ \pm}$. Then,

(1)

$$
f\left(L^{+}\right)=f\left(L^{-}\right)=0
$$

(2)

$$
\lim _{x \rightarrow \pm \infty} u(x, y)=L^{ \pm}
$$

for every $y \geq 0$; 
(3)

$$
\left\|u-L^{ \pm}\right\|_{L^{\infty}\left(B_{R}^{+}(x, 0)\right)} \rightarrow 0 \quad \text { as } x \rightarrow \pm \infty
$$

(4)

$$
\left\|\nabla_{x} u\right\|_{L^{\infty}\left(B_{R}^{+}(x, 0)\right)}+\left\|y^{a} \frac{\partial u}{\partial y}\right\|_{L^{\infty}\left(B_{R}^{+}(x, 0)\right)} \rightarrow 0 \text { as } x \rightarrow \pm \infty .
$$

Proof Our proof uses the invariance of the problem under periodic translations in $x$ and a compactness argument.

Denote $u^{n}(x, y)=u(x+n, y)$ for $n \in \mathbb{Z}$. Since $b$ is 1 -periodic, $u^{n}$ still satisfies the equations

$$
\begin{cases}-\operatorname{div}\left(y^{a} \nabla u^{n}\right)=0 & \text { in } \mathbb{R}_{+}^{2} \\ -\lim _{y \downarrow 0^{+}} y^{a} \frac{\partial u^{n}}{\partial y}=\frac{1}{d_{s}} b(x) f\left(u^{n}(x, 0)\right) & \text { on } \partial \mathbb{R}_{+}^{2}\end{cases}
$$

By regularity results in [2] and [5], we see that up to a subsequence,

$$
\begin{aligned}
& u^{n} \rightarrow u^{ \pm \infty} \quad \text { in } C_{\mathrm{loc}}^{0}\left(\overline{\mathbb{R}_{+}^{2}}\right), \\
& \nabla_{x} u^{n} \rightarrow \nabla_{x} u^{ \pm \infty} \quad \text { in } C_{\mathrm{loc}}^{0}\left(\overline{\mathbb{R}_{+}^{2}}\right), \\
& y^{a} \frac{\partial u^{n}}{\partial y} \rightarrow y^{a} \frac{\partial u^{\infty}}{\partial y} \quad \text { in } C_{\mathrm{loc}}^{0}\left(\overline{\mathbb{R}_{+}^{2}}\right)
\end{aligned}
$$

as $n \rightarrow \pm \infty$. Then $u^{ \pm \infty}$ solves the equations

$$
\begin{cases}-\operatorname{div}\left(y^{a} \nabla u^{ \pm \infty}\right)=0 & \text { in } \mathbb{R}_{+}^{2}, \\ -\lim _{y \downarrow 0^{+}} y^{a} \frac{\partial u^{ \pm \infty}}{\partial y}=\frac{1}{d_{s}} b(x) f\left(u^{ \pm \infty}\right) & \text { on } \partial \mathbb{R}_{+}^{2}\end{cases}
$$

and it follows that $u^{ \pm \infty}(x, 0) \equiv L^{ \pm}$for every $x \in \mathbb{R}$.

Consider the Dirichlet problem

$$
\begin{cases}-\operatorname{div}\left(y^{a} \nabla u^{ \pm \infty}\right)=0 & \text { in } \mathbb{R}_{+}^{2} \\ u^{ \pm \infty}(x, 0) \equiv L^{ \pm} & \text {on } \partial \mathbb{R}_{+}^{2}\end{cases}
$$

$u^{ \pm \infty} \equiv L^{ \pm}$is the unique solution of (2.8) by Corollary 3.5 in [2]. As a consequence, (2.2) and (2.5) are obvious.

The following lemma is a necessary condition for a local minimizer of the energy functional $\mathcal{E}$.

Lemma 2.2 Let $u$ be a local minimizer of the energy functional $\mathcal{E}$ under perturbations in $[-1,1]$. That is, for any bounded Lipschitz domain $\Omega \subset \mathbb{R}_{+}^{2}$ and for any $\xi \in H^{1}\left(y^{a}, \Omega\right)$ having compact support in $\Omega \cup \partial^{0} \Omega$ such that $u+\xi \in[-1,1]$,

$$
\mathcal{E}(u, \Omega) \leq \mathcal{E}(u+\xi, \Omega) .
$$


Let

$$
\lim _{x \rightarrow \pm \infty} u(x, 0)= \pm 1
$$

Then

$$
G(1)=G(-1) \leq G(\tau) \text { for all } \tau \in(-1,1) \text {. }
$$

Proof To show (2.10), it is sufficient to prove that $G(1) \leq G(\tau)$ and $G(-1) \leq G(\tau)$ for all $\tau \in$ $[-1,1]$. Suppose $G\left(\tau_{0}\right)<G(1)$ for some point $\tau_{0} \in[-1,1]$ by contradiction. For simplicity, assume that $G\left(\tau_{0}\right)=0$ by adding a constant.

By (2.9),

$$
\liminf _{l \rightarrow+\infty} \mathcal{E}\left(u, B_{R}^{+}(l, 0)\right) \geq \liminf _{l \rightarrow+\infty} \int_{\partial^{0} B_{R}^{+}(l, 0)} b(x) G(u(x, 0)) \geq 2 \underline{b} \varepsilon R
$$

for some $\varepsilon>0$.

Let $\xi_{R}$ be a cut-off function with values in $[0,1]$,

$$
\xi_{R}= \begin{cases}1 & \text { in } B_{(1-\eta) R} \\ 0 & \text { in } \mathbb{R}^{n+1} \backslash B_{R}\end{cases}
$$

where $\eta \in(0,1)$ will be specified later, and $\left|\nabla \xi_{R}\right| \leq \frac{1}{\eta R}$.

Define $\xi_{R, l}(x, y)=\xi_{R}(x-l, y)$. Let $w=\tau_{0} \xi_{R, l}+\left(1-\xi_{R, l}\right) u$, then $w=u$ on $\partial^{+} B_{R}^{+}(l, 0)$ and $w \equiv \tau_{0}$ in $B_{(1-\eta) R}^{+}(l, 0)$. We have

$$
\begin{aligned}
\limsup _{l \rightarrow+\infty} \mathcal{E}_{B_{R}^{+}(l, 0)}(w)= & \limsup _{l \rightarrow+\infty}\left\{d_{s} \int_{B_{R}^{+}(l, 0)} \frac{y^{a}}{2}\left|\left(1-\xi_{R, l}\right) \nabla u+\left(\tau_{0}-u\right) \nabla \xi_{R, l}\right|^{2}\right. \\
& \left.+\int_{\partial^{0} B_{R}^{+}(l, 0)} b\left(x_{1}\right) G(w)\right\} \\
\leq & 2 d_{s} \int_{B_{R}^{+}} y^{a}\left|\nabla \xi_{R, l}\right|^{2}+2 \bar{b} \max _{[-1,1]} G \cdot \eta R \\
\leq & \frac{C d_{s} R^{a}}{\eta^{2}}+2 \bar{b} \max _{[-1,1]} G \cdot \eta R .
\end{aligned}
$$

We use (2.5) in the first inequality above.

Having chosen $\eta=\frac{\underline{b} \varepsilon}{2 \bar{b} \max _{[-1,1]} G}$, by (2.11) and (2.12),

$$
\limsup _{l \rightarrow+\infty} \mathcal{E}\left(w, B_{R}^{+}(l, 0)\right)<\liminf _{l \rightarrow+\infty} \mathcal{E}\left(u, B_{R}^{+}(l, 0)\right)
$$

for large $R>1$. This contradiction leads to $G(1) \leq G(\tau)$ for all $\tau \in[-1,1]$. By the same discussion, $G(-1) \leq G(\tau)$ for all $\tau \in[-1,1]$. Thus we complete the proof.

As in [2], we construct a Hamiltonian equality which will be used in the proof of Theorem 1.3. For this purpose a lemma is in order, for whose proof see Lemma 5.1 in [2]. 
Lemma 2.3 Let $u \in L^{\infty}\left(\mathbb{R}_{+}^{2}\right)$ be a solution of (1.2). Then for every $x \in \mathbb{R}, \int_{0}^{\infty} y^{a}|\nabla u|^{2} d y<\infty$. In addition, the integral can be differentiated with respect to $x \in \mathbb{R}$ under the integral sign. We have

$$
\lim _{M \rightarrow+\infty} \int_{M}^{\infty} y^{a}|\nabla u|^{2} d y=0
$$

uniformly in $x \in \mathbb{R}$. If $u$ is a layer solution of (1.2),

$$
\lim _{|x| \rightarrow+\infty} \int_{0}^{\infty} y^{a}|\nabla u|^{2} d y=0 .
$$

Proposition 2.1 (Hamiltonian equality) Let $u$ be a layer solution of (1.2) for $a \in\left(-1, \frac{1}{2}\right)$, i.e.,

$$
\begin{cases}-\operatorname{div}\left(y^{a} \nabla u\right)=0 & \text { in } \mathbb{R}_{+}^{2}, \\ -\lim _{y \downarrow 0^{+}} y^{a} \frac{\partial u}{\partial y}=\frac{1}{d_{s}} b(x) f(u) & \text { on } \partial \mathbb{R}_{+}^{2}, \\ u(x, 0) \rightarrow \pm 1 & \text { as } x \rightarrow \infty\end{cases}
$$

For every $x \in \mathbb{R}$, the Hamiltonian equality holds:

$$
\begin{aligned}
d_{s} \int_{0}^{\infty} \frac{y^{a}}{2}\left\{u_{x}^{2}-u_{y}^{2}\right\} d y= & b(x)\{G(u(x, 0))-G(1)\} \\
& +\int_{x}^{\infty} b^{\prime}(t)\{G(u(t, 0))-G(1)\} d t .
\end{aligned}
$$

As a consequence,

$$
\int_{-\infty}^{+\infty} b^{\prime}(x)\{G(u(x, 0))-G(1)\} d x=0 .
$$

Proof We note that the integral in (2.16) is well defined since $s=\frac{1-a}{2} \in\left(\frac{1}{4}, 1\right)$ and

$$
G(u(x, 0))-G(1)=\frac{G^{\prime \prime}(t)}{2}(u(x, 0)-1)^{2}=O\left(|x|^{-4 s}\right) \quad \text { as }|x| \rightarrow \infty,
$$

where $t$ is some point between $u(x, 0)$ and 1 .

By Lemma 2.3, the left integral in (2.15) can be differentiated with respect to $x$,

$$
\begin{aligned}
\frac{d}{d x} d_{s} \int_{0}^{\infty} \frac{y^{a}}{2}\left\{u_{x}^{2}-u_{y}^{2}\right\} d y & =d_{s} \int_{0}^{\infty} y^{a}\left\{u_{x} u_{x x}-u_{y} u_{y x}\right\} d y \\
& =d_{s} \int_{0}^{\infty} y^{a}\left\{u_{x x}+u_{y y}+\frac{a}{y} u_{y}\right\} u_{x} d y+d_{s} \lim _{y \rightarrow 0^{+}} y^{a} u_{y} u_{x} \\
& =-b(x) f(u(x, 0)) u_{x}(x, 0) .
\end{aligned}
$$

In the second equality above we use the fact that $\lim _{y \rightarrow \infty} y^{a} u_{y} u_{x}=0$ (see [2]). We have

$$
\begin{aligned}
& \frac{d}{d x}\left\{b(x)(G(u(x, 0))-G(1))+\int_{x}^{+\infty} b^{\prime}(t)(G(u(t, 0))-G(1)) d x\right\} \\
& \quad=-b(x) f(u(x, 0)) u_{x}(x, 0) .
\end{aligned}
$$


Thus,

$$
\begin{aligned}
d_{s} \int_{0}^{\infty} \frac{y^{a}}{2}\left\{u_{x}^{2}-u_{y}^{2}\right\} d y \equiv & b(x)\{G(u(x, 0))-G(1)\} \\
& +\int_{x}^{+\infty} b^{\prime}(t)\{G(u(t, 0))-G(1)\} d t+C .
\end{aligned}
$$

Let $x \rightarrow+\infty$, the left of (2.17) converging to zero by (2.14); thus $C=0$ and (2.15) is proved. Letting $x \rightarrow-\infty$, (2.16) is also obtained.

To study asymptotic estimates of layer solutions of (1.1), we recall an explicit layer solution of the unperturbed problem (1.3).

Lemma 2.4 ([3], Theorem 3.1) Let $s \in(0,1)$. For every $t>0$, the $C^{\infty}$ function

$$
v_{s}^{t}(x)=\operatorname{sign}(x) \frac{2}{\pi} \int_{0}^{\infty} \frac{\sin (z)}{z} e^{-t\left(\frac{z}{|x|}\right)^{2 s}} d z
$$

is the layer solution to the fractional equation

$$
\left(-\partial_{x x}\right)^{s} v_{s}^{t}=f_{s}^{t}\left(v_{s}^{t}\right) \quad \text { in } \mathbb{R}
$$

for a nonlinearity $f_{s}^{t} \in C^{1}([-1,1])$ which is odd and twice differentiable in $[-1,1]$ and which satisfies

$$
f_{s}^{t}(0)=f_{s}^{t}(1)=0, \quad f_{s}^{t}>0 \text { in }(0,1), \quad\left(f_{s}^{t}\right)^{\prime}( \pm 1)=-\frac{1}{t} .
$$

In addition, the following limits exist:

$$
\lim _{|x| \rightarrow \infty}|x|^{1+2 s}\left(\partial_{x} v_{s}^{t}\right)(x)=t \frac{4 s}{\pi} \sin (\pi s) \Gamma(2 s)>0
$$

and, as a consequence,

$$
\lim _{x \rightarrow \pm \infty}|x|^{2 s}\left|\left(v_{s}^{t}\right)(x) \mp 1\right|=t \frac{2}{\pi} \sin (\pi s) \Gamma(2 s)>0 .
$$

\section{Existence and asymptotic estimates}

To prove the existence of layer solutions, we introduce a Liouville result where $a \leq 0$ is required. This is the reason why we restrict ourselves to the case $s \geq \frac{1}{2}$ in Theorem 1.1.

Proposition 3.1 Let $a \leq 0$. Suppose $u$ is a bounded nonnegative function which satisfies weakly the problem

$$
\begin{cases}-\operatorname{div}\left(y^{a} \nabla u\right) \leq 0 & \text { in } \mathbb{R}_{+}^{2}, \\ -\lim _{y \downarrow 0^{+}} y^{a} \frac{\partial u}{\partial y} \leq 0 & \text { on } \partial \mathbb{R}_{+}^{2} .\end{cases}
$$

Then $u \equiv C$ a.e. in $\mathbb{R}_{+}^{2}$. 
Proof Since $a \leq 0, R^{a} \leq 1$ for $R>1$. Let $\xi$ be a smooth function with values in [0,1], $\xi=1$ in $B_{R}$ and $\xi=0$ outside of $B_{2 R},|\nabla \xi| \leq C R^{-1}$. Multiplying (3.1) with $u \xi^{2}$ and integrating by parts, we have that

$$
\begin{aligned}
\int_{B_{R}^{+}} y^{a}|\nabla u|^{2} & \leq \int_{B_{2 R}^{+}} y^{a}|\nabla u|^{2} \xi^{2} \leq 2 \int_{\mathbb{R}_{+}^{2}} y^{a} \xi u|\nabla u||\nabla \xi| \\
& \leq 2\left\{\int_{B_{2 R}^{+} \backslash B_{R}^{+}} y^{a}|\nabla u|^{2} \xi^{2}\right\}^{\frac{1}{2}}\left\{\int_{B_{2 R}^{+} \backslash B_{R}^{+}} y^{a}|\nabla \xi|^{2} u^{2}\right\}^{\frac{1}{2}} \\
& \leq C\left\{\int_{B_{2 R}^{+} \backslash B_{R}^{+}} y^{a}|\nabla u|^{2} \xi^{2}\right\}^{\frac{1}{2}}\left(R R^{1+a} R^{-2}\right)^{\frac{1}{2}} \\
& \leq C\left\{\int_{B_{2 R}^{+} \backslash B_{R}^{+}} y^{a}|\nabla u|^{2} \xi^{2}\right\}^{\frac{1}{2}} .
\end{aligned}
$$

Thus $\int_{\mathbb{R}_{+}^{2}} y^{a}|\nabla u|^{2} \leq C$ for some constant $C$ independent of $R$. Let $R \rightarrow \infty$, $\int_{B_{2 R}^{+} \backslash B_{R}^{+}} y^{a}|\nabla u|^{2} \xi^{2} \rightarrow 0$. We deduce that $\int_{\mathbb{R}_{+}^{2}} y^{a}|\nabla u|^{2}=0$ and $u \equiv C$ a.e. in $\mathbb{R}_{+}^{2}$.

Next we prove an existence result about the local minimizer of $\mathcal{E}$.

Lemma 3.1 Let $\Omega \subset \mathbb{R}_{+}^{2}$ be a bounded Lipschitz domain. Let $w_{0} \in C^{0}(\bar{\Omega}) \cap H^{1}\left(y^{a}, \Omega\right)$ be a given function with $\left|w_{0}\right| \leq 1$; $b$ is a bounded positive function.

Suppose that

$$
f(1) \leq 0 \leq f(-1)
$$

the energy functional $\mathcal{E}(u, \Omega)$ admits a minimizer $u \in \mathcal{C}_{w_{0}, a}=\left\{w \in H^{1}\left(y^{a}, \Omega\right),-1 \leq w \leq\right.$ 1 a.e. in $\Omega, w=w_{0}$ on $\partial^{+} \Omega$ in the weak sense $\}$, which solves weakly

$$
\begin{cases}-\operatorname{div}\left(y^{a} \nabla u\right)=0 & \text { in } \Omega, \\ -\lim _{y \downarrow 0^{+}} y^{a} \frac{\partial u}{\partial y}=\frac{1}{d_{s}} b(x) f(u(x, 0)) & \text { on } \partial^{0} \Omega, \\ u=w_{0} & \text { on } \partial^{+} \Omega .\end{cases}
$$

Moreover, $u$ is a stable solution of (3.2), i.e.,

$$
d_{s} \int_{\Omega} y^{a}|\nabla \xi|^{2} d x d y-\int_{\partial^{0} \Omega} b(x) f^{\prime}(u) \xi^{2} d x \geq 0
$$

for every $\xi \in H^{1}\left(\Omega, y^{a}\right)$ such that $\xi \equiv 0$ on $\partial^{+} \Omega$ in the weak sense.

Proof Consider the set $H_{w_{0}, a}(\Omega)=\left\{w \in H^{1}\left(y^{a}, \Omega\right), w \equiv w_{0}\right.$ on $\partial^{+} \Omega$ in the weak sense $\}$ $\mathcal{C}_{w_{0}, a}, H_{w_{0}, a}(\Omega) \neq \emptyset$ since $w_{0} \in H_{w_{0}, a}(\Omega)$. Denote

$$
\widetilde{f}= \begin{cases}f(1) & \text { if } t \geq 1 \\ f & \text { if }-1<t<1 \\ f(-1) & \text { if } t \leq-1\end{cases}
$$

and $\widetilde{G}=-\int_{0}^{u} \widetilde{f}$. Up to an additive constant, $\widetilde{G}=G$ in $[-1,1]$. 
Consider the energy functional

$$
\widetilde{\mathcal{E}}(u, \Omega)=d_{s} \int_{\Omega} \frac{y^{a}}{2}|\nabla u|^{2} d x d y+\int_{\partial^{0} \Omega} b(x) \widetilde{G}(u(x, 0)) d x .
$$

If $\widetilde{\mathcal{E}}$ has an absolute minimizer $u$ in $\mathcal{C}_{w_{0}, a}(\Omega)$, the statement of Lemma 3.1 is proved.

For every function $w \in H_{w_{0}, a}(\Omega), w-w_{0} \in H^{1}\left(y^{a}, \Omega\right)$ and vanishes on $\partial^{+} \Omega$ in the weak sense. We can extend $w-w_{0}$ in $\mathbb{R}_{+}^{2}$ by zeroes outside of $\Omega$ and $w-w_{0} \in H^{1}\left(y^{a}, \mathbb{R}_{+}^{2}\right)$. By the trace theorem and the Sobolev imbedding theorem (see [7, 11, 12]),

$$
H^{1}\left(y^{a}, \mathbb{R}_{+}^{2}\right) \hookrightarrow L^{p}(\mathbb{R})
$$

for $p=\frac{2}{1-2 s}$ if $s<\frac{1}{2}$ or for any $1 \leq p<\infty$ if $s \geq \frac{1}{2}$. Moreover, $H^{1}\left(y^{a}, \mathbb{R}_{+}^{2}\right) \hookrightarrow \hookrightarrow L^{2}\left(\partial^{0} \Omega\right)$.

Since $\widetilde{G}$ has linear growth at infinity, $\widetilde{\mathcal{E}}$ is well defined, bounded below and coercive in $H_{w_{0}, a}$. There exists an absolute minimizer $u \in H_{w_{0}, a}$. By the first order variation, we have

$$
\begin{cases}-\operatorname{div}\left(y^{a} \nabla u\right)=0 & \text { in } \Omega, \\ -\lim _{y \downarrow 0^{+}} y^{a} \frac{\partial u}{\partial y}=\frac{1}{d_{s}} b\left(x_{1}\right) \tilde{f}(u(x, 0)) & \text { on } \partial^{0} \Omega\end{cases}
$$

Multiply $(u-1)^{+}$with (3.5) and integrate in $\Omega$,

$$
d_{s} \int_{\Omega} y^{a}\left|\nabla(u-1)^{+}\right|^{2} d x d y-\int_{\partial^{0} \Omega} b(x) f(1)(u-1)^{+} d x=0 .
$$

Since $f(1) \leq 0, \int_{\Omega} y^{a}\left|\nabla(u-1)^{+}\right|^{2} d x d y \leq 0$. Thus $(u-1)^{+} \equiv 0$ a.e. in $\Omega$, i.e., $u \leq 1$ a.e. in $\Omega$. Similarly we also get $u \geq-1$ a.e. in $\Omega$. Hence $u \in \mathcal{C}_{w_{0}, a}(\Omega)$. (3.2) follows from (3.5), and (3.3) comes from the second order variation of $\mathcal{E}$.

Remark 3.1 Suppose that $b$ is an even function, $f$ and $w_{0}$ are odd with respect to $x$, with a slight modification we can also show that there is an odd minimizer in the admissible set $\left\{w \in \mathcal{C}_{w_{0}, a} \mid w(-x, y)=-w(x, y)\right.$ for every $\left.y \geq 0\right\}$.

Now we start to show the existence of layer solutions of (1.2).

Theorem 3.1 Let $s \geq \frac{1}{2}$. Let $b \in\left(C^{1, \gamma} \cap L^{\infty}\right)(\mathbb{R})$ and $f \in C^{1, \gamma}(\mathbb{R})(0<\gamma<1)$ :

(a) $b: \mathbb{R} \rightarrow \mathbb{R}$ is an even positive function, $b(x+1)=b(x) \forall x \in \mathbb{R}$,

(b) $f(-\tau)=-f(\tau)$ for any $\tau \in[-1,1], f(-1)=f(1)=f(0)=0, f>0$ in $(0,1)$ and $f<0$ in $(-1,0)$.

Then there exists a layer solution $u$ of $(1.2)$ in $\mathbb{R}_{+}^{2}$ :

$$
\begin{cases}-\operatorname{div}\left(y^{a} \nabla u\right)=0 & \text { in } \mathbb{R}_{+}^{2}, \\ -\lim _{y \downarrow 0^{+}} y^{a} \frac{\partial u}{\partial y}=\frac{1}{d_{s}} b(x) f(u(x, 0)) & \text { on } \partial \mathbb{R}_{+}^{2},\end{cases}
$$

which is odd with respect to $x$, i.e., $u(-x, y)=-u(x, y)$, and, for every $y \geq 0$,

$$
\lim _{x \rightarrow \pm \infty} u(x, y)= \pm 1
$$


Furthermore, $u$ is a local minimizer of the energy functional $\mathcal{E}$ under odd perturbations in $[-1,1]$, and it is stable in the sense that

$$
d_{s} \int_{\mathbb{R}_{+}^{2}} y^{a}|\nabla \xi|^{2} d x d y-\int_{\mathbb{R}} b(x) f^{\prime}(u(x, 0)) \xi^{2} d x \geq 0
$$

for every function $\xi \in C^{1}\left(\overline{\mathbb{R}_{+}^{2}}\right)$ with compact support in $\overline{\mathbb{R}_{+}^{2}}, \xi(-x, y)=-\xi(x, y)$ and $u+\xi \in$ $[-1,1]$.

Proof The proof is divided into three parts. For simplicity, we make $G(1)=G(-1)=0$ by adding a constant.

Step 1. We show that there exists a solution with values in $[-1,1]$ of $(3.6)$ which is odd with respect to the variable $x$ for every $y \geq 0$.

Let $Q_{R}=[-R, R] \times[0, R]$ and $w_{0}=\frac{\arctan x}{\arctan R}$. Define the admissible set

$$
\mathcal{C}_{w_{0}, a, o}=\left\{w \in C_{w_{0}, a}\left(Q_{R}\right), \forall y \geq 0, w(-x, y)=-w(x, y)\right\} .
$$

By Remark 3.1, there is a minimizer $u_{R}$ in $\mathcal{C}_{w_{0}, a, o}$,

$$
\begin{cases}-\operatorname{div}\left(y^{a} \nabla u_{R}\right)=0 & \text { in } Q_{R}, \\ -\lim _{y \downarrow 0^{+}} y^{a} \frac{\partial u_{R}}{\partial y}=\frac{1}{d_{s}} b(x) f\left(u_{R}(x, 0)\right) & \text { on } \partial^{0} Q_{R}, \\ u_{R}=w_{0} & \text { on } \partial^{+} Q_{R} .\end{cases}
$$

Define

$$
u_{R}:= \begin{cases}u_{R}(-x, y) & \text { if } u_{R}(x, y)<0 \text { and } x>0 \\ u_{R}(x, y) & \text { if } u_{R}(x, y) \geq 0 \text { and } x>0\end{cases}
$$

and $u_{R}(x, y):=-u_{R}(-x, y)$ for $x \leq 0$. Thus $u_{R} \geq 0$ for $x>0$ and $y \geq 0$. Obviously $u_{R}$ is still a minimizer of $\mathcal{E}\left(\cdot, Q_{R}\right)$.

By the regularity results in [2], $u_{R}, \nabla_{x} u_{R}, y^{a} \frac{\partial u_{R}}{\partial y} \in C^{\beta}\left(Q_{R}\right)$ for some $0<\beta<1$ and the continuous module is uniform bounded. Up to a subsequence, $u_{R} \rightarrow u,\left(u_{R}\right)_{x} \rightarrow u_{x}$ and $y^{a} \frac{\partial u_{R}}{\partial y} \rightarrow y^{a} \frac{\partial u}{\partial y}$ in $C^{0}\left(\overline{B_{s}^{+}}\right)$as $R \rightarrow \infty$ for all $R>s+2$. By the canonical diagonal procedure, $u$ solves

$$
\begin{cases}-\operatorname{div}\left(y^{a} \nabla u\right)=0 & \text { in } \mathbb{R}_{+}^{2}, \\ -\lim _{y \downarrow 0^{+}} y^{a} \frac{\partial u}{\partial y}=\frac{1}{d_{s}} b(x) f(u(x, 0)) & \text { on } \partial \mathbb{R}_{+}^{2}, \\ u(-x, y)=-u(x, y) & \text { in } \overline{\mathbb{R}_{+}^{2}}\end{cases}
$$

and by the Hopf maximum principle $-1<u<1$.

Step 2. We show that there exists at least a subsequence $x_{n} \rightarrow \infty$ such that $u\left(x_{n}, 0\right) \rightarrow 1$.

First we claim that $u$ is a local minimizer under odd perturbations in $[-1,1]$. That is,

$$
\mathcal{E}(u, \Omega) \leq \mathcal{E}(w, \Omega)
$$

for any $\Omega \subset \mathbb{R}_{+}^{2}$ and for any odd function $w \in H^{1}\left(y^{a}, \Omega\right)$ with $|w| \leq 1$ and $w=u$ on $\partial^{+} \Omega$ in the weak sense. 
Let $\xi \in C_{c}^{1}\left(B_{s}^{+} \cup \partial^{0} B_{s}^{+}\right)$is odd with respect to $x$ for every $y \geq 0$ and $u_{R}+\xi \in[-1,1]$. Since $-1<u_{R}<1, u_{R}+(1-\epsilon) \xi \in(-1,1)$ for $\epsilon \in(0,1)$. We have

$$
\mathcal{E}\left(u_{R}, B_{s}^{+}\right) \leq \mathcal{E}\left(u_{R}+(1-\epsilon) \xi, B_{s}^{+}\right) \quad \text { for } R>s+2 .
$$

Let $R \rightarrow \infty$, and

$$
\mathcal{E}\left(u, B_{s}^{+}\right) \leq \mathcal{E}\left(u+(1-\epsilon) \xi, B_{s}^{+}\right)
$$

for every $s>0$ and $u+(1-\epsilon) \xi \in[-1,1]$. Our claim is proved.

$$
\text { If } w(-x, y)=-w(x, y) \text {, }
$$

$$
\mathcal{E}\left(w, B_{s}^{+}\right)=2 \mathcal{E}\left(w, B_{s}^{++}\right)=2\left\{d_{s} \int_{B_{s}^{++}} \frac{y^{a}}{2}|\nabla w|^{2} d x d y+\int_{\partial^{0} B_{s}^{++}} b(x) G(w) d x\right\}
$$

where $B_{s}^{++}=\left\{(x, y) \in B_{s}^{+}, x>0, y \geq 0\right\}$. Therefore $u$ is also a local minimizer of $\mathcal{E}$ in $\mathbb{R}_{++}^{n+1}=$ $\left\{(x, y) \in \mathbb{R}_{+}^{2}, x>0, y \geq 0\right\}$ with perturbations in $[-1,1]$, i.e.,

$$
\mathcal{E}(u, \Omega) \leq \mathcal{E}(w, \Omega)
$$

for any $\Omega \subset \mathbb{R}_{++}^{2}$ and for any $w \in H^{1}\left(y^{a}, \Omega\right)$ with $|w| \leq 1$ and $w=u$ on $\partial^{+} \Omega$ in weak sense.

Suppose $u\left(x_{n}, 0\right) \nrightarrow 1$ for any sequence $x_{n} \rightarrow \infty$ by contradiction. $|u(x, 0)|<1-\epsilon$ for some $0<\epsilon<1$ and $x \in \mathbb{R}$. Hence $0 \leq u(x, y)<1-\epsilon$ for all $x>0$ and $y \geq 0$ by the fact that $u(\cdot, y)=P_{s}(\cdot, y) * u(\cdot, 0)$.

Let $R>1$. Let $\varphi_{R}$ be a cut-off function with values 1 in $B_{(1-\eta) R}^{+}$and zeroes outside of $B_{R}^{+}$, $\left|\nabla \varphi_{R}\right| \leq \frac{C}{\eta R}$ for some $0<\eta<1$ determined later.

Denote $\varphi_{R}=\varphi_{R}(|(x-l, y)|)$. Let $w=1 \cdot \varphi_{R}+\left(1-\varphi_{R}\right) u \in H^{1}\left(y^{a}, B_{R}^{+}(l, 0)\right), w \equiv u$ on $\partial^{+} B_{R}(l, 0)$. For $l>R$,

$$
\begin{aligned}
\mathcal{E}\left(w, B_{R}^{+}(l, 0)\right)= & d_{s} \int_{B_{R}^{+}(l, 0)} \frac{y^{a}}{2}\left|\left(1-\varphi_{R}\right) \nabla u+(1-u) \nabla \varphi_{R}\right|^{2} d x d y+\int_{\partial^{0} B_{R}^{+}(l, 0)} b(x) G(w) d x \\
\leq & d_{s} \int_{B_{R}^{+}(l, 0)} \frac{y^{a}}{2}|\nabla u|^{2} d x d y+d_{s} \int_{B_{R}^{+}(l, 0)} \frac{y^{a}}{2}\left|\nabla \varphi_{R}\right|^{2} d x d y \\
& +d_{s}\left\{\int_{B_{R}^{+}(l, 0)} y^{a}|\nabla u|^{2} d x d y\right\}^{\frac{1}{2}}\left\{\int_{B_{R}^{+}} y^{a}\left|\nabla \varphi_{R}\right|^{2} d x d y\right\}^{\frac{1}{2}} \\
& +\int_{\partial^{0}\left(B_{R}^{+} \backslash B_{(1-\eta) R}^{+}\right)} b(x) G(w) d x \\
\leq & d_{s} \int_{B_{R}^{+}(l, 0)} \frac{y^{a}}{2}|\nabla u|^{2} d x d y+\left(C \eta^{-2} R^{-2} R R^{1+a}\right) \\
& +\left\{C R\left[\int_{1}^{R} y^{a} y^{-2} d y+\int_{0}^{1}\left(y^{a}+y^{-a}\right) d y\right]\right\}^{\frac{1}{2}}\left(C \eta^{-2} R^{a}\right)^{\frac{1}{2}} \\
& +2 \bar{b} \max _{[0,1]} G \cdot \eta R \\
\leq & d_{s} \int_{B_{R}^{+}(l, 0)} \frac{y^{a}}{2}|\nabla u|^{2} d x d y+C \eta^{-2} R^{a}+C \eta^{-1} R^{\frac{1+a}{2}}+2 \bar{b} \max _{[0,1]} G \cdot \eta R .
\end{aligned}
$$


Here the constant $C$ does not depend on $R$, we use the gradient estimates (see [2]) in the second line from the bottom.

On the other hand,

$$
\mathcal{E}\left(u, B_{R}^{+}(l, 0)\right) \geq d_{s} \int_{B_{R}^{+}(l, 0)} \frac{y^{a}}{2}|\nabla u|^{2} d x d y+2 \underline{b} \min _{[0,1-\epsilon]} G \cdot R .
$$

Choose $\eta=\frac{\underline{b} \min _{[0,1-\epsilon]} G}{2 \bar{b} \max _{[0,1]} G}, \mathcal{E}\left(u, B_{R}^{+}(l, 0)\right)>\mathcal{E}\left(w, B_{R}^{+}(l, 0)\right)$ for large $R$. This contradiction leads to the result that there exists at least a sequence $x_{n} \rightarrow \infty$ such that $u\left(x_{n}, 0\right) \rightarrow 1$.

Step 3. We show that $u$ is the layer solution, i.e., $\lim _{x \rightarrow \pm \infty} u(x, 0)= \pm 1$.

Let $u^{n}(x, y)=u(x+n, y)$ and $n \in \mathbb{Z}^{+}$. By the regularity results [2], up to a subsequence,

$$
\begin{aligned}
& u^{n} \rightarrow u^{\infty} \quad \text { in } C_{\mathrm{loc}}^{0}\left(\overline{\mathbb{R}_{+}^{2}}\right), \\
& u_{x}^{n} \rightarrow u_{x}^{\infty} \quad \text { in } C_{\mathrm{loc}}^{0}\left(\overline{\mathbb{R}_{+}^{2}}\right), \\
& y^{a} \frac{\partial u^{n}}{\partial y} \rightarrow y^{a} \frac{\partial u^{\infty}}{\partial y} \quad \text { in } C_{\mathrm{loc}}^{0}\left(\overline{\mathbb{R}_{+}^{2}}\right)
\end{aligned}
$$

as $n \rightarrow \infty$.

$$
\begin{cases}-\operatorname{div}\left(y^{a} \nabla u^{\infty}\right)=0 & \text { in } \mathbb{R}_{+}^{2}, \\ -\lim _{y \downarrow 0^{+}} y^{a} \frac{\partial u^{\infty}}{\partial y}=\frac{1}{d_{s}} b(x) f\left(u^{\infty}(x, 0)\right) & \text { on } \partial \mathbb{R}_{+}^{2}, \\ 0 \leq u^{\infty} \leq 1 & \text { in } \mathbb{R}_{+}^{2} .\end{cases}
$$

Define $\widetilde{u}=1-u^{\infty}$, we have

$$
\begin{cases}-\operatorname{div}\left(y^{a} \nabla \widetilde{u}\right)=0 & \text { in } \mathbb{R}_{+}^{2}, \\ -\lim _{y \downarrow 0^{+}} y^{a} \frac{\partial \widetilde{u}}{\partial y}=-\frac{1}{d_{s}} b(x) f\left(u^{\infty}(x, 0)\right) \leq 0 & \text { on } \partial \mathbb{R}_{+}^{2} \\ 0 \leq \widetilde{u} \leq 1 & \text { in } \mathbb{R}_{+}^{2}\end{cases}
$$

$\widetilde{u} \equiv C$ by Proposition $3.1, f\left(u^{\infty}(x, 0)\right)=f(C) \equiv 0$ and $u^{\infty} \equiv 0$ or 1 . Thus $u^{\infty} \equiv 1$ by step 2. That is, $u \rightarrow 1$ as $x \rightarrow \infty . u \rightarrow-1$ as $x \rightarrow-\infty$ is achieved by odd symmetry.

$u$ is the desired layer solution.

Proof of Theorem 1.1 It follows from Theorem 3.1; for the regularity of $v$ see [2].

Lastly we give asymptotic estimates for layer solutions of (1.1) as $|x| \rightarrow \infty$.

Proof of Theorem 1.2 Let $v$ be a layer solution of (1.1),

$$
\left\{\begin{array}{l}
\left(-\partial_{x x}\right)^{s} v(x)=b(x) f(v(x)) \text { in } \mathbb{R} \\
\lim _{x \rightarrow \pm \infty} v= \pm 1
\end{array}\right.
$$

Then

$$
\left(-\partial_{x x}\right)^{s}(1-v)-b(x) f^{\prime}\left(\xi_{1}\right)(1-v)=0 \quad \text { in } \mathbb{R}
$$

where $\xi_{1}$ is some point between $v(x)$ and 1 . 
Consider the layer solution $v_{s}^{t}$ of the unperturbed problem in Lemma 2.4,

$$
\left(-\partial_{x x}\right)^{s}\left(1-v_{s}^{t}\right)-\left(f_{s}^{t}\right)^{\prime}\left(\xi_{2}\right)\left(1-v_{s}^{t}\right)=0 \quad \text { in } \mathbb{R}
$$

with $\xi_{2}$ is some point between $v_{s}^{t}(x)$ and 1 .

Since $-\left(f_{s}^{t}\right)^{\prime}(1)=\frac{1}{t}$, choose $t$ large enough such that $\frac{2}{t}<-\underline{b} f^{\prime}(1)$ and choose $x_{0} \in \mathbb{R}$ such that $-\left(f_{s}^{t}\right)^{\prime}\left(\xi_{2}\right)<\frac{2}{t}<-\underline{b} f^{\prime}\left(\xi_{1}\right)$ for all $x>x_{0}$.

Choose $C>0$ such that $C\left(1-v_{s}^{t}\right)>1-v$ in $\left(-\infty, x_{0}\right]$, which can be done since $v_{s}^{t}, v \rightarrow-1$ as $x \rightarrow-\infty$.

Define

$$
d(x)= \begin{cases}\frac{2}{t} & \text { in }\left(x_{0},+\infty\right) \\ \frac{C f_{s}^{t}\left(v_{s}^{t}\right)-b(x) f(v)}{C\left(1-v_{s}^{t}\right)-(1-v)} & \text { in }\left(-\infty, x_{0}\right]\end{cases}
$$

$d(x) \in L^{\infty}$. We have

$$
\begin{cases}\left(-\partial_{x x}\right)^{s}\left\{C\left(1-v_{s}^{t}\right)-(1-v)\right\}+d(x)\left\{C\left(1-v_{s}^{t}\right)-(1-v)\right\} \geq 0 & \text { in } \mathbb{R} \\ C\left(1-v_{s}^{t}\right)-(1-v)>0 & \text { in }\left(-\infty, x_{0}\right]\end{cases}
$$

Obviously, if $\inf _{\mathbb{R}}\left\{C\left(1-v_{s}^{t}\right)-(1-v)\right\}<0$, it is achieved at some point $\underline{x} \in\left(x_{0},+\infty\right)$. Since $d>0$ in $\left(x_{0},+\infty\right),\left(-\partial_{x x}\right)^{s}\left\{C\left(1-v_{s}^{t}\right)-(1-v)\right\}(\underline{x}) \geq 0$ from the first inequality of (3.16), which contradicts with the fact that

$$
\begin{aligned}
& \left(-\partial_{x x}\right)^{s}\left\{C\left(1-v_{s}^{t}\right)-(1-v)\right\}(\underline{x}) \\
& \quad=\int_{R} \frac{\left\{C\left(1-v_{s}^{t}\right)-(1-v)\right\}(\underline{x})-\left\{C\left(1-v_{s}^{t}\right)-(1-v)\right\}(y)}{|\underline{x}-y|^{1+2 s}} d y<0 .
\end{aligned}
$$

Therefore $(1-v) \leq C\left(1-v_{s}^{t}\right)$ for $C>0$ given from above.

On the other hand, choose small $t>0$ such that $-\bar{b} f^{\prime}(1)<\frac{1}{2 t}$ and choose $x^{0} \in \mathbb{R}$ such that $-\bar{b} f^{\prime}\left(\xi_{1}\right)<\frac{1}{2 t}<-\left(f_{s}^{t}\right)^{\prime}\left(\xi_{2}\right)$ for all $x>x^{0}$. Choose $c>0$ such that $c\left(1-v_{s}^{t}\right)<1-v$ in $\left(-\infty, x^{0}\right]$.

Define

$$
\tilde{d}(x)= \begin{cases}\frac{1}{2 t} & \text { in }\left(x^{0},+\infty\right) \\ \frac{b(x) f(v)-c f_{s}^{t}\left(v_{s}^{t}\right)}{(1-v)-c\left(1-v_{s}^{s}\right)} & \text { in }\left(-\infty, x^{0}\right]\end{cases}
$$

and obviously $\tilde{d}(x) \in L^{\infty}$.

Then,

$$
\begin{cases}\left(-\partial_{x x}\right)^{s}\left\{(1-v)-c\left(1-v_{s}^{t}\right)\right\}+\widetilde{d}(x)\left\{(1-v)-c\left(1-v_{s}^{t}\right)\right\} \geq 0 & \text { in } \mathbb{R} \\ (1-v)-c\left(1-v_{s}^{t}\right)>0 & \text { in }\left(-\infty, x^{0}\right] .\end{cases}
$$

If $\inf _{R}\left\{(1-v)-c\left(1-v_{s}^{t}\right)\right\}<0$, it is only achieved at some point $\bar{x} \in\left(x^{0},+\infty\right)$. Since $\widetilde{d}>$ 0 in $\left(x^{0},+\infty\right),\left(-\partial_{x x}\right)^{s}\left\{(1-v)-c\left(1-v_{s}^{t}\right)\right\}(\bar{x}) \geq 0$ from the first inequality of (3.17), which contradicts the fact that $\left(-\partial_{x x}\right)^{s}\left\{(1-v)-c\left(1-v_{s}^{t}\right)\right\}(\bar{x})<0$. Thus $c\left(1-v_{s}^{t}\right) \leq(1-v)$ for some $0<c<C$ given from above. 
Therefore,

$$
c x^{-2 s} \leq|1-v| \leq C x^{-2 s} \text { for } x>1
$$

by Lemma 2.4 . Similarly,

$$
c|x|^{-2 s} \leq|1+v| \leq C|x|^{-2 s} \quad \text { for } x<-1 .
$$

Here $c$ and $C$ maybe different from above.

\section{Asymptotic as $s \uparrow 1$}

In this section we prove Theorem 1.3, which consists of two lemmas.

Lemma 4.1 Let $\left\{v^{s_{k}}\right\}$ be a sequence of layer solutions of (1.1) in Theorem 1.1. Then there exists a subsequence denoted again by $\left\{v^{s k}\right\}$, converging locally uniformly to $v^{1}$ which solves the local elliptic equation

$$
-v_{x x}^{1}(x)=b(x) f\left(v^{1}\right) \quad \text { in } \mathbb{R}
$$

Proof Consider $u_{a_{k}}$, the $s$-extension of $v^{s_{k}}$, which solves

$$
\begin{cases}-\operatorname{div}\left(y^{a_{k}} \nabla u_{a_{k}}\right)=0 & \text { in } \mathbb{R}_{+}^{2}, \\ -\left(1+a_{k}\right) \lim _{y \downarrow 0^{+}} y^{a_{k}} \partial_{y} u_{a_{k}}=C_{a_{k}} b(x) f\left(u_{a_{k}}(x, 0)\right) & \text { on } \partial \mathbb{R}_{+}^{2},\end{cases}
$$

where $a_{k}=1-2 s_{k}$ and $C_{a_{k}}=\frac{1+a_{k}}{d_{s_{k}}}=\frac{2\left(1-s_{k}\right)}{d_{s_{k}}}$. Obviously $a_{k} \downarrow-1$ as $s_{k} \uparrow 1$.

Let $\xi \in C_{c}^{1}\left(\overline{\mathbb{R}_{+}^{2}}\right)$. Multiplying (4.2) with $\xi$ and integrating in $\mathbb{R}_{+}^{2}$,

$$
\left(1+a_{k}\right) \int_{\mathbb{R}_{+}^{2}} y^{a_{k}} \nabla u_{a_{k}} \nabla \xi d x d y-C_{a_{k}} \int_{\mathbb{R}} b(x) f\left(u_{a_{k}}(x, 0)\right) \xi d x=0 .
$$

Choose $\xi(x, y)=\xi_{1}(x) \xi_{2}(y), \xi_{1} \in C_{c}^{1}(\mathbb{R})$ and $\xi_{2}$ is a cut-off function which equals 1 in $[0,1]$ and 0 in $[2, \infty),\left|\xi_{2}^{\prime}\right| \leq C$ for some constant $C>0$. Thus (4.3) can be rewritten as

$$
\begin{aligned}
(1 & \left.+a_{k}\right) \int_{\mathbb{R}_{+}^{2}} y^{a_{k}}\left\{\xi_{1}^{\prime}(x) \xi_{2}(y) \partial_{x} u_{a_{k}}+\xi_{1}(x) \xi_{2}^{\prime}(y) \partial_{y} u_{a_{k}}\right\} d x d y \\
& =C_{a_{k}} \int_{\mathbb{R}} b(x) f\left(u_{a_{k}}(x, 0)\right) \xi_{1}(x) d x
\end{aligned}
$$

By the regularity results in [2], the continuous module does not depend on $s$ for $s>s_{0}>\frac{1}{2}$. Up to a subsequence,

$$
\begin{aligned}
& u_{a_{k}} \rightarrow u_{-1} \quad \text { in } C_{\mathrm{loc}}^{0}\left(\overline{\mathbb{R}_{+}^{2}}\right), \\
& \left(u_{a_{k}}\right)_{x} \rightarrow\left(u_{-1}\right)_{x} \text { in } C_{\mathrm{loc}}^{0}\left(\overline{\mathbb{R}_{+}^{2}}\right)
\end{aligned}
$$

and

$$
C_{a_{k}}=\frac{2\left(1-s_{k}\right)}{d_{s_{k}}}=\frac{2\left(1-s_{k}\right)}{2^{2 s_{k}-1} \frac{\Gamma\left(s_{k}\right)}{\Gamma\left(1-s_{k}\right)}} \rightarrow 1
$$


as $s_{k} \uparrow 1$ (or equivalently $a_{k} \downarrow-1$ ). Then

$$
C_{a_{k}} \int_{\mathbb{R}} b(x) f\left(u_{a_{k}}(x, 0)\right) \xi_{1} d x \rightarrow \int_{\mathbb{R}} b(x) f\left(u_{-1}(x, 0)\right) \xi_{1} d x \quad \text { as } a_{k} \downarrow-1 .
$$

For the first integral in (4.4), we consider

$$
\begin{aligned}
& \left(1+a_{k}\right) \int_{0}^{\infty} y^{a_{k}} \xi_{2}(y) \partial_{x} u_{a_{k}} d y \\
& =\left(1+a_{k}\right) \int_{0}^{\delta} y^{a_{k}} \xi_{2}(y)\left\{\partial_{x} u_{a_{k}}-u_{-1}^{\prime}(x)\right\} d y \\
& +\left(1+a_{k}\right) \int_{0}^{\delta} y^{a_{k}} \xi_{2}(y) u_{-1}^{\prime}(x) d y+\left(1+a_{k}\right) \int_{\delta}^{\infty} y^{a_{k}} \xi_{2}(y) \partial_{x} u_{a_{k}} d y \\
& =I_{1}+I_{2}+I_{3} \text {, } \\
& \left|I_{1}\right| \leq\left(1+a_{k}\right) \int_{0}^{\delta} y^{a_{k}} \xi_{2}(y)\left|\partial_{x} u_{a_{k}}-u_{-1}^{\prime}(x)\right| d y \leq \epsilon \delta^{1+a_{k}}
\end{aligned}
$$

for $0<\delta<1$ and small $\epsilon>0$. Here we use the fact that $\partial_{x} u_{a_{k}} \rightarrow u_{-1}^{\prime}(x)$ locally uniformly in $\overline{\mathbb{R}_{+}^{2}}$. We have

$$
I_{2}=u_{-1}^{\prime}(x)\left(1+a_{k}\right) \int_{0}^{\delta} y^{a_{k}} d y=\delta^{1+a_{k}} u_{-1}^{\prime} \rightarrow u_{-1}^{\prime} \quad \text { as } a_{k} \downarrow-1 .
$$

Since $\left|\nabla u_{a_{k}}\right| \leq \frac{C}{y}$ for $y>0$ and $C$ independent of $a_{k}$ (see [2]),

$$
\left|I_{3}\right| \leq C\left(1+a_{k}\right) \int_{\delta}^{\infty} y^{a_{k}-1} d y=C \frac{1+a_{k}}{a_{k}} \delta^{a_{k}} \rightarrow 0 \quad \text { as } a_{k} \downarrow-1 .
$$

By (4.6)-(4.9),

$$
\left(1+a_{k}\right) \int_{0}^{\infty} y^{a_{k}} \xi_{2}(y) \partial_{x} u_{a_{k}} d y \rightarrow u_{-1}^{\prime}
$$

and

$$
\begin{gathered}
\left(1+a_{k}\right) \int_{\mathbb{R}_{+}^{2}} y^{a_{k}} \xi_{1}^{\prime}(x) \xi_{2}(y) \partial_{x} u_{a_{k}} d x d y \rightarrow \int_{\mathbb{R}} \xi_{1}^{\prime}(x) u_{-1}^{\prime} d x \\
\left|\left(1+a_{k}\right) \int_{\mathbb{R}_{+}^{2}} y^{a_{k}} \xi_{1}(x) \xi_{2}^{\prime}(y) \partial_{y} u_{a_{k}} d x d y\right| \\
\leq \int_{\mathbb{R}}\left|\xi_{1}(x)\right| d x\left(1+a_{k}\right) \int_{1}^{2} y^{a_{k}}\left|\xi_{2}^{\prime}(y)\right|\left|\partial_{y} u_{a_{k}}\right| d y \\
\leq C\left(1+a_{k}\right) \int_{1}^{2} y^{a_{k}-1} d y=C \frac{1+a_{k}}{a_{k}}\left(2^{a_{k}}-1\right) \rightarrow 0
\end{gathered}
$$

as $a_{k} \downarrow-1$.

Therefore, by (4.4), (4.5), (4.10), and (4.11),

$$
\int_{\mathbb{R}} u_{-1}^{\prime}(x) \xi_{1}^{\prime}(x) d x=\int_{\mathbb{R}} b(x) f\left(u_{-1}(x)\right) \xi_{1}(x) d x
$$


That is,

$$
-v_{x x}^{1}=b(x) f\left(v^{1}\right)
$$

in the weak sense $\left(u_{-1}=v^{1}\right)$. By the regularity theory of elliptic equations, $v^{1}$ is also a classical solution of (4.13).

Lemma 4.2 $v^{1}$ is also a layer solution of (4.1), i.e., $v^{1} \rightarrow \pm 1$ as $x \pm \infty$.

Proof Claim 1. $v^{1}$ is a local minimizer in $(0, \infty)$ under perturbations in $[-1,1]$. That is,

$$
\mathcal{F}\left(v^{1}, I\right) \leq \mathcal{F}\left(v^{1}+\xi_{1}, I\right)
$$

for any bounded open interval $I \subset(0, \infty)$ and for any $\xi_{1} \in C_{0}^{1}(I)$ such that $\left|v^{1}+\xi_{1}\right| \leq 1$, where

$$
\mathcal{F}(w, I):=\int_{I}\left\{\frac{\left|w_{x}\right|^{2}}{2}+b(x) G(w)\right\} d x \quad \text { for every } w \in H^{1}(I) .
$$

Indeed, for the test function $\xi$ in Lemma 4.1 with the additional property that $\left|u_{a_{k}}+\xi\right| \leq$ 1 , we have

$$
\begin{aligned}
0 \leq & E\left(u_{a_{k}}+(1-\epsilon) \xi, I \times[0, R]\right)-E\left(u_{a_{k}}, I \times[0, R]\right) \\
= & \frac{1+a_{k}}{2} \int_{I \times[0, R]} y^{a_{k}}\left|\nabla\left(u_{a_{k}}+(1-\epsilon) \xi\right)\right|^{2} d x d y+C_{a_{k}} \int_{I} b(x) G\left(u_{a_{k}}+(1-\epsilon) \xi\right) d x \\
& -\frac{1+a_{k}}{2} \int_{I \times[0, R]} y^{a_{k}}\left|\nabla u_{a_{k}}\right|^{2} d x d y-C_{a_{k}} \int_{I} b(x) G\left(u_{a_{k}}\right) d x \\
= & \frac{1+a_{k}}{2} \int_{I \times[0, R]} y^{a_{k}}\left|\partial_{x} u_{a_{k}}+(1-\epsilon) \xi_{1}^{\prime}(x) \xi_{2}(y)\right|^{2} d x d y \\
& -\frac{1+a_{k}}{2} \int_{I \times[0, R]} y^{a_{k}}\left|\partial_{x} u_{a_{k}}\right|^{2} d x d y \\
& +\left(1+a_{k}\right) \int_{I \times[0, R]} y^{a_{k}} \partial_{y} u_{a_{k}}(1-\epsilon) \xi_{1}(x) \xi_{2}^{\prime}(y) d x d y \\
& +\frac{1+a_{k}}{2} \int_{I \times[0, R]} y^{a_{k}}\left((1-\epsilon) \xi_{1}(x) \xi_{2}^{\prime}(y)\right)^{2} d x d y \\
& +C_{a_{k}} \int_{I} b(x) G\left(u_{a_{k}}+(1-\epsilon) \xi_{1}(x)\right) d x-C_{a_{k}} \int_{I} b(x) G\left(u_{a_{k}}\right) d x .
\end{aligned}
$$

As in the discussions in Lemma 4.1, let $a_{k} \downarrow-1$, and we have

$$
\begin{aligned}
& \frac{1+a_{k}}{2} \int_{I \times[0, R]} y^{a_{k}}\left(\partial_{x} u_{a_{k}}\right)^{2} d x d y \rightarrow \int_{I} \frac{\left(u_{-1}^{\prime}\right)^{2}}{2} d x, \\
& \left(1+a_{k}\right) \int_{I \times[0, R]} y^{a_{k}} \partial_{x} u_{a_{k}}(1-\epsilon) \xi_{1}^{\prime}(x) \xi_{2}(y) d x d y \rightarrow \int_{I} u_{-1}^{\prime}(x)(1-\epsilon) \xi_{1}^{\prime}(x) d x,
\end{aligned}
$$




$$
\begin{aligned}
& \frac{1+a_{k}}{2} \int_{I \times[0, R]} y^{a_{k}}\left((1-\epsilon) \xi_{1}^{\prime}(x) \xi_{2}(y)\right)^{2} d x d y \\
& \quad=\frac{1}{2} \int_{I}(1-\epsilon)^{2}\left(\xi_{1}^{\prime}(x)\right)^{2} d x\left\{\int_{0}^{1}\left(1+a_{k}\right) y^{a_{k}} d y+\int_{1}^{R}\left(1+a_{k}\right) y^{a_{k}}\left(\xi_{2}(y)\right)^{2} d y\right\} \\
& \quad \rightarrow \frac{1}{2} \int_{I}\left((1-\epsilon) \xi_{1}^{\prime}(x)\right)^{2} d x
\end{aligned}
$$

By (4.16)-(4.18),

$$
\begin{aligned}
& \frac{1+a_{k}}{2} \int_{I \times[0, R]} y^{a_{k}}\left(\partial_{x} u_{a_{k}}+(1-\epsilon) \xi_{1}^{\prime}(x) \xi_{2}(y)\right)^{2} d x d y \\
& -\frac{1+a_{k}}{2} \int_{I \times[0, R]} y^{a_{k}}\left(\partial_{x} u_{a_{k}}\right)^{2} d x d y \\
& \rightarrow \frac{1}{2} \int_{I}\left(u_{-1}^{\prime}(x)+(1-\epsilon) \xi_{1}^{\prime}(x)\right)^{2} d x-\frac{1}{2} \int_{I}\left(u_{-1}^{\prime}(x)\right)^{2} d x, \\
& \left(1+a_{k}\right) \int_{I \times[0, R]} y^{a_{k}} \partial_{y} u_{a_{k}} \xi_{1}(x) \xi_{2}^{\prime}(y) d x d y \\
& =\left(1+a_{k}\right) \int_{I \times[1,2]} y^{a_{k}} \partial_{y} u_{a_{k}} \xi_{1}(x) \xi_{2}^{\prime}(y) d x d y \\
& \leq C\left(1+a_{k}\right) \int_{1}^{2} y^{a_{k}-1} d y \\
& =\frac{C\left(1+a_{k}\right)}{a_{k}}\left\{2^{a_{k}}-1\right\} \rightarrow 0 \quad \text { as } a_{k} \downarrow-1, \\
& \frac{\left(1+a_{k}\right)}{2} \int_{I} \int_{0}^{R} y^{a_{k}}\left(\xi_{1}(x) \xi_{2}^{\prime}(y)\right)^{2} d x d y=\frac{\left(1+a_{k}\right)}{2} \int_{I} \int_{1}^{2} y^{a_{k}}\left(\xi_{1}(x) \xi_{2}^{\prime}(y)\right)^{2} d x d y \\
& \leq C\left(1+a_{k}\right) \int_{1}^{2} y^{a_{k}} d y \\
& =C\left(2^{a_{k}+1}-1\right) \rightarrow 0 \quad \text { as } a_{k} \downarrow-1, \\
& C_{a_{k}} \int_{I} b(x) G\left(u_{a_{k}}+(1-\epsilon) \xi_{1}(x)\right) d x-C_{a_{k}} \int_{I} b(x) G\left(u_{a_{k}}\right) d x \\
& \rightarrow \int_{I} b(x) G\left(u_{-1}+(1-\epsilon) \xi_{1}(x)\right) d x-\int_{I} b(x) G\left(u_{-1}\right) d x .
\end{aligned}
$$

By (4.15), (4.19)-(4.22), our claim is proved.

Claim 2. $v^{1} \rightarrow 1$ as $x \rightarrow \infty$.

Define $v^{1, n}(x)=v^{1}(x+n)$ for $n \in \mathbb{Z}^{+}$, up to a subsequence, $v^{1, n} \rightarrow v^{1, \infty}$ in $C_{\text {loc }}^{2}$ as $n \rightarrow \infty$,

$$
\left\{\begin{array}{l}
-v_{x x}^{1, \infty}(x)=b(x) f\left(v^{1, \infty}(x)\right), \quad x \in \mathbb{R} \\
0 \leq v^{1, \infty} \leq 1
\end{array}\right.
$$

Since $f \geq 0$ and $b>0,-v_{x x}^{1, \infty} \geq 0$ in $\mathbb{R}$ and $v^{1, \infty} \equiv 0$ or 1 .

We show that $v^{1} \rightarrow 0$ or 1 as $x \rightarrow \infty$. Indeed, if there are two sequences $\left\{x_{n}\right\}$ and $\left\{y_{n}\right\}$ such that $v^{1}\left(x_{n}\right) \rightarrow 0$ and $v^{1}\left(y_{n}\right) \rightarrow 1$ as $n \rightarrow \infty$, there must exist $z_{n} \in\left(x_{n}, y_{n}\right)$ such that $v^{1}\left(z_{n}\right)=\frac{1}{2}$.

Denote $\widetilde{v}_{n}^{1}(x)=v^{1}\left(x+\left[z_{n}\right]\right)$ where $\left[z_{n}\right]$ is the integer part of $z_{n} \cdot \widetilde{v}_{n}^{1}\left(z_{n}-\left[z_{n}\right]\right)=v^{1}\left(z_{n}\right)=\frac{1}{2}$ and up to a subsequence $\widetilde{v}_{n}^{1} \rightarrow \widetilde{v}_{\infty}^{1}$ in $C_{\text {loc }}^{2}, \widetilde{v}_{\infty}^{1}$ solves equation (4.23). Therefore $\widetilde{v}_{\infty}^{1} \equiv 0$ or 1 . 
For the above subsequence, there is a subsubsequence such that $z_{n}-\left[z_{n}\right] \rightarrow z^{*} \in[0,1]$ as $n \rightarrow \infty$ and $\widetilde{v}_{\infty}^{1}\left(z^{*}\right)=\frac{1}{2}$. This contradiction verifies $v^{1} \rightarrow 0$ or 1 as $x \rightarrow \infty$.

To check $v^{1} \rightarrow 1$ as $x \rightarrow \infty$, suppose that $v^{1} \rightarrow 0$ as $x \rightarrow \infty$ by contradiction. Then,

$$
\liminf _{l \rightarrow+\infty} \mathcal{F}\left(v^{1},(l-R, l+R)\right)=\liminf _{l \rightarrow+\infty} \int_{l-R}^{l+R}\left\{\frac{\left|v^{1}\right|^{2}}{2}+b(x) G\left(v^{1}\right)\right\} d x \geq 2 \underline{b} R \epsilon
$$

for some $\epsilon>0$.

Let $\xi \in C_{0}^{1}(l-R, l+R), \xi=1$ if $|x-l|<(1-\eta) R$ and $\xi=0$ if $|x-l|>R$ where $\eta$ will be determined later, $\left|\xi^{\prime}\right| \leq \frac{1}{\eta R}$. Define $w=1 \cdot \xi+(1-\xi) v^{1}$, then $w(l \pm R)=v^{1}(l \pm R)$. We have

$$
\begin{aligned}
& \limsup _{l \rightarrow+\infty} \mathcal{F}(w,(l-R, l+R)) \\
& \quad=\limsup _{l \rightarrow+\infty} \int_{l-R}^{l+R}\left(\frac{1}{2}\left|(1-\xi) v_{x}^{1}+\left(1-v^{1}\right) \xi_{x}\right|^{2}+b(x) G\left(1 \cdot \xi+(1-\xi) v^{1}\right)\right) d x \\
& \quad \leq \int_{l-R}^{l+R} \xi_{x}^{2} d x+\bar{b} \max _{[-1,1]} G \cdot 2 \eta R \\
& \quad \leq \frac{1}{\eta^{2} R}+\bar{b} \max _{[-1,1]} G \cdot 2 \eta R .
\end{aligned}
$$

Choose $\eta=\frac{\epsilon \underline{b}}{2 \bar{b} \max _{[-1,1]} G}$,

$$
\limsup _{l \rightarrow+\infty} \mathcal{F}(w,(l-R, l+R))<\liminf _{l \rightarrow+\infty} \mathcal{F}\left(v^{1},(l-R, l+R)\right)
$$

for $R>1$ large enough. Therefore $v^{1} \rightarrow 1$ as $x \rightarrow \infty$, by odd symmetry, $v^{1} \rightarrow-1$ as $x \rightarrow-\infty$, i.e., $v^{1}$ is a layer solution of the local elliptic equation (4.13).

By the Hamiltonian equality (2.15),

$$
\begin{aligned}
b(x) & \left\{G\left(v^{1}(x)\right)-G(1)\right\}+\int_{x}^{+\infty} b^{\prime}(t)\left\{G\left(v^{1}(t)\right)-G(1)\right\} d t \\
= & \frac{1}{2}\left(v_{x}^{1}\right)^{2}=\lim _{a_{k} \downarrow-1}\left(1+a_{k}\right) \int_{0}^{\infty} \frac{y^{a_{k}}}{2}\left(\partial_{x} u_{a_{k}}\right)^{2} \\
= & \lim _{a_{k} \downarrow-1}\left(1+a_{k}\right) \int_{0}^{\infty} \frac{y^{a_{k}}}{2}\left(\partial_{y} u_{a_{k}}\right)^{2} \\
& +\lim _{a_{k} \downarrow-1} C_{a_{k}} b(x)\left\{G\left(u_{a_{k}}(x, 0)\right)-G(1)\right\} \\
& +\lim _{a_{k} \downarrow-1} C_{a_{k}} \int_{x}^{\infty} b^{\prime}(t)\left\{G\left(u_{a_{k}}(t, 0)\right)-G(1)\right\} d t .
\end{aligned}
$$

Therefore,

$$
\begin{aligned}
\lim _{a \downarrow-1}(1+a) \int_{0}^{\infty} \frac{y^{a}}{2}\left(\partial_{y} u_{a}\right)^{2}= & \int_{x}^{+\infty} b^{\prime}(t)\left\{G\left(v^{1}(t)\right)-G(1)\right\} d t \\
& -\lim _{a_{k} \downarrow-1} C_{a_{k}} \int_{x}^{\infty} b^{\prime}(t)\left\{G\left(u_{a_{k}}(t, 0)\right)-G(1)\right\} d t .
\end{aligned}
$$

Proof of Theorem 1.3 It follows from Lemmas 4.1 and 4.2. 
Competing interests

The author declares that she has no competing interests.

\section{Author's contributions}

The author read and approved the final manuscript.

\section{Acknowledgements}

This research has been supported by National Natural Science Foundation of China (Grant No. 11371128).

Received: 4 November 2013 Accepted: 4 February 2014 Published: 17 Feb 2014

\section{References}

1. Cabré, X, Solà-Morales, J: Layer solutions in a half-space for boundary reactions. Commun. Pure Appl. Math. 58(12), 1678-1732 (2005)

2. Cabré, $X$, Sire, Y: Nonlinear equations for fractional Laplacians I: regularity, maximum principles, and Hamiltonian estimates. Ann. Inst. Henri Poincaré, Anal. Non Linéaire. (2013). doi:10.1016/j.anihpc.2013.02.001

3. Cabré, $X$, Sire, Y: Nonlinear equations for fractional Laplacian II: existence, uniqueness, and qualitative properties of solutions. arXiv:1111.0796 (2011)

4. Caffarelli, L, Silvestre, L: An extension problem related to the fractional Laplacian. Commun. Partial Differ. Equ. 32(8), $1245-1260$ (2007)

5. Fabes, EB, Kenig, CE, Serapioni, RP: The local regularity of solutions of degenerate elliptic equations. Commun. Stat. Theory Methods 7(1), 77-116 (1982)

6. Palatucci, G, Savin, O, Valdinoci, E: Local and global minimizers for a variational energy involving a fractional norm. Ann. Mat. Pura Appl. 192(4), 673-718 (2013)

7. Nekvinda, A: Characterization of traces of the weighted Sobolev space $W^{1, p}\left(\Omega, d_{M}^{\varepsilon}\right)$ on $M$. Czechoslov. Math. J. 43(4), 695-711 (1993)

8. Alama, S, Bronsard, L, Gui, C: Stationary layered solutions in $\mathbb{R}^{2}$ for an Allen-Cahn systems with multiple well potential. Calc. Var. Partial Differ. Equ. 5(4), 359-390 (1997)

9. Ghoussoub, N, Gui, C: On a conjecture of De Giorgi and some related problems. Math. Ann. 311, 481-491 (1998)

10. Ghoussoub, N, Gui, C: On De Giorgi's conjecture in dimensions 4 and 5. Ann. Math. 157(1), 313-334 (2003)

11. Adams, RA: Sobolev Spaces. Pure and Applied Mathematics, vol. 65. Academic Press, New York (1975)

12. Gilbarg, D, Trudinger, NS: Elliptic Partial Differential Equations of Second Order. Springer, Berlin (1997)

10.1186/1687-2770-2014-41

Cite this article as: $\mathrm{Hu}$ : Layer solutions for a class of semilinear elliptic equations involving fractional Laplacians. Boundary Value Problems 2014, 2014:41

\section{Submit your manuscript to a SpringerOpen ${ }^{0}$ journal and benefit from:}

- Convenient online submission

- Rigorous peer review

- Immediate publication on acceptance

Open access: articles freely available online

High visibility within the field

- Retaining the copyright to your article 